\title{
OralViewer: 3D Demonstration of Dental Surgeries for Patient Education with Oral Cavity Reconstruction from a 2D Panoramic X-ray
}

\author{
Yuan Liang \\ University of California, Los Angeles \\ liangyuandg@g.ucla.edu \\ Zhujun Fang \\ University of California, Los Angeles \\ floraqa@g.ucla.edu \\ Yiting Shao \\ University of California, Los Angeles \\ shaobruin0011@ucla.edu
}

\author{
Liang Qiu \\ University of California, Los Angeles \\ liangqiu@g.ucla.edu
}

Dezhan Tu

University of California, Los Angeles

dezhantu@gmail.com

Kun Wang

University of California, Los Angeles

wangk@ucla.edu

Lei He

University of California, Los Angeles

lhe@ee.ucla.edu

\author{
Tiancheng Lu \\ University of Pittsburgh \\ til62@pitt.edu \\ Jiawei Yang
}

University of California, Los Angeles jiawei118@g.ucla.edu

Xiang 'Anthony' Chen

University of California, Los Angeles

xac@ucla.edu
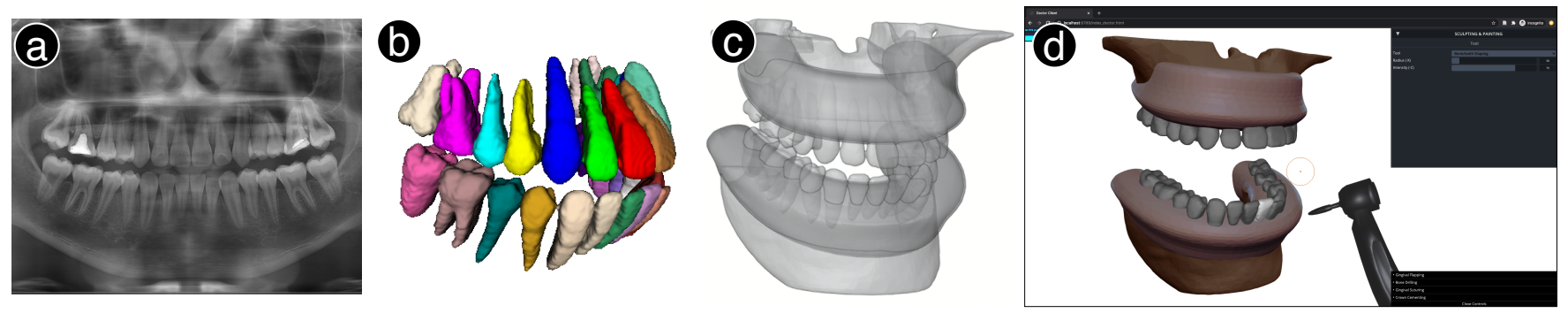

Figure 1: Overview of OralViewer. The system takes a patient's 2D X-ray as input (a), and reconstructs the 3D teeth structure (b) with a novel deep learning model. The system then generates the complete oral cavity model (c) by registering the pre-defined models of jaw bone and gum to the dental arch curve. Finally, a dentist can demonstrate the forthcoming surgeries to a patient by animating the steps with our virtual dental instruments (d).

\begin{abstract}
Patient's understanding on forthcoming dental surgeries is required by patient-centered care and helps reduce anxiety. Due to the complexity of dental surgeries and the patient-dentist expertise gap, conventional techniques of patient education are usually not effective for explaining surgical steps. In this paper, we present OralViewer-the first interactive application that enables dentist's demonstration of dental surgeries in 3D to promote patients' understanding. OralViewer takes a single 2D panoramic dental X-ray to reconstruct patient-specific 3D teeth structures, which are then assembled with registered gum and jaw bone models for complete
\end{abstract}

\section{(c) (7) (8)}

This work is licensed under a Creative Commons Attribution-NonCommercial International 4.0 License.

IUI '21, April 14-17, 2021, College Station, TX, USA

(C) 2021 Copyright held by the owner/author(s).

ACM ISBN 978-1-4503-8017-1/21/04.

https://doi.org/10.1145/3397481.3450695 oral cavity modeling. During the demonstration, OralViewer enables dentists to show surgery steps with virtual dental instruments that can animate effects on a 3D model in real-time. A technical evaluation shows that our deep learning model achieves a mean Intersection over Union (IoU) of 0.771 for $3 \mathrm{D}$ teeth reconstruction. A patient study with 12 participants shows OralViewer can improve patients' understanding of surgeries. A preliminary expert study with 3 board-certified dentists further verifies the clinical validity of our system.

\section{CCS CONCEPTS}

- Human-centered computing $\rightarrow$ Human computer interaction (HCI).

\section{KEYWORDS}

deep learning, 3D visualization, patient education 
ACM Reference Format:

Yuan Liang, Liang Qiu, Tiancheng Lu, Zhujun Fang, Dezhan Tu, Jiawei Yang, Yiting Shao, Kun Wang, Xiang 'Anthony' Chen, and Lei He. 2021. OralViewer: 3D Demonstration of Dental Surgeries for Patient Education with Oral Cavity Reconstruction from a 2D Panoramic X-ray. In 26th International Conference on Intelligent User Interfaces (IUI '21), April 1417, 2021, College Station, TX, USA. ACM, New York, NY, USA, 11 pages. https://doi.org/10.1145/3397481.3450695

\section{INTRODUCTION}

Patient-dentist communication is a core requirement of patientcentered care [5]. According to [31, 40, 44, 48], patients who understand their dentists are more likely to follow medication schedules, feel satisfied about treatments and have better oral health outcomes. Moreover, many patients about to undergo dental surgeries can experience anxiety - up to every fourth adult reported dental fear $[3,36]$. To manage the dental fear, one solution is to unveil the surgical steps to decrease patients' fear of the unknown $[4,8,21]$. Previous studies have observed shorter duration of surgery [15], lower level of post-operative pain [22], and smoother recovery [23] with a reduced dental fear.

Dental surgeries can be complex and challenging to explain [18]. Currently, dentists mostly perform pre-operative patient education via verbal explanation, possibly with the aid of hand-drawn diagrams [10], audiovisual slides [12] and video clips [23]. Meanwhile, the recent advent of 3D demonstration, which illustrates complex procedures with dynamic visuals in 3D, has shown an increasing potential in patient education since it is more intuitive and complete than verbal description and static images. Indeed, existing studies have explored the 3D demonstration for cardiac surgeries [11, 29], condylar reconstruction [54] and pancreatectomy [16] to assist the pre-operative communications. However, to the best of our knowledge, 3D demonstration for dental clinics has been unexplored.

To fill this gap, we present OralViewer, a web-based system to enable dentists to virtually demonstrate dental surgeries on 3D oral cavity models for patient education. To inform the design of OralViewer, we interviewed three dentists and elicited key system requirements from a clinical point of view: (i) providing a patientspecific 3D teeth model, (ii) modeling the complete oral cavity of teeth, gums and jaw bones, , and (iii) demonstrating surgery steps using simple operations. In terms of 3D oral cavity modeling, OralViewer goes beyond existing work [7, 28, 49] that extracts a patient's anatomy models from high-cost 3D scanning, e.g., computerized tomography (CT) scans. Instead, we enable the generation of $3 \mathrm{D}$ models from a single 2D panoramic X-ray image with a novel deep learning model. This approach lowers the barrier of obtaining a 3D model as the 2D panoramic X-ray is the most common modality in dentistry and the only required imaging for many dental surgeries [24]. In terms of surgery demonstration, OralViewer implements virtual dental instruments that are simple to operate with a mouse and illustrative with real-time effects on oral cavity models for patients to understand. Figure 1 shows the workflow: the system first takes a patient's panoramic X-ray to generate the $3 \mathrm{D}$ teeth structure $(\mathrm{a} \rightarrow \mathrm{b})$; then pre-defined gum and jaw bone models are registered to the dental arch, and assembled with the teeth structure for the complete oral cavity model (c); finally, a dentist operates virtual dental instruments on the reconstructed oral cavity to demonstrate a forthcoming surgery to a patient (d).

We validate OralViewer for the demonstration of two common dental surgeries: crown lengthening and apicoectomy. Note that the design and implementation of OralViewer (e.g., the 3D reconstruction process and virtual operation techniques) are expected to generalize to other dental surgeries as well. These two surgeries were selected because each of them requires multiple steps and various commonly-used dental instruments, thus are ideal for testing the capability of OralViewer. We conducted three evaluations: (i) A technical evaluation of 3D teeth reconstruction from 2D panoramic X-ray shows our model achieves an average IoU of $0.771 \pm 0.062$. (ii) A study with 12 patient indicates that our system leads to patients' improved understanding of dental surgeries. (iii) A preliminary expert study with 3 board-certificated dentists suggests that the demonstration using our system is clinically valid, can improve the efficiency of patient education, yet there remain areas for improvement in the operation of virtual tools.

Contributions of this paper include:

- OralViewer - the first solution that enables 3D demonstration of dental surgeries for patient education;

- 3D modeling - the first 3D reconstruction technique of oral cavity from a single $2 \mathrm{D}$ panoramic X-ray;

- Evaluation - a patient study and an expert study validate the feasibility and usability of educating patients with 3D simulative demonstration.

\section{BACKGROUND}

In this section, we briefly introduce the common steps in typical apicoectomy and crown lengthening surgeries. Descriptions with figures are included in Appendix, while [18] gives more detailed information.

Apicoectomy is the removal of the root tip and surrounding tissues of a tooth with periapical inflammation. A dentist first performs a periodontal flap - incises and flaps the gum tissue for unveiling the underlying bone structure with scalpels. Next, the apex is exposed by creating an peripheral opening on the buccal (jaw) bone with a round bur and a steady stream of saline solution. After that, the inflamed root tip can be resected with a handpiece, followed by filling material into the tooth cavity created to seal it. Then, bone grafting materials can be injected into the jaw bone hole for rehabilitation, and finally the periodontal flap being repositioned and sutured.

Crown Lengthening can be applied for restoring cavities and tooth fractures that happen below the gum tissue. To start, a dentist incises and flaps the gum tissue to unveil the target structure. Next, the jaw bone's height at a surrounding area is often reduced with a bur, in order to support the repositioned gum in a lower position below the cavity/fracture. Then, the cavity is removed (or fracture shaped) with a handpiece, and restored with grafting materials. For better protecting the tooth, the restored crown is sometimes further shaped (with handpieces), and cemented with an artifact crown.

\section{RELATED WORK}

In this section, we first review existing work on 3D surgical visualization and simulation. Then, we summarize the 3D-based 
Computer-Aided Design (CAD) technologies for dentistry. We also include a review on the deep learning algorithms for $3 \mathrm{D}$ reconstruction from a single $2 \mathrm{D}$ view.

\subsection{Surgical Visualization and Simulation}

Conventional techniques of delivering education to patients through verbal instructions may not be effective to explain surgical procedures due to the educational barriers between the patients and the clinicians. Researches have shown 3D anatomy visualization can improve the patients' understanding of surgeries, where example systems include those for abdominal [28], cardiac system [35, 51], and more [49]. Moreover, with the advent of computer graphics, interactive manipulation of virtual 3D models has shown to help patients acquire a more satisfactory level of knowledge $[16,17,37]$. Recently, studies [41, 42] have also incorporated virtual reality (VR) to enable more intuitive anatomy viewing.

In comparison, OralViewer is different from all the aforementioned work from two aspects. First, all the 3D anatomy models used are either captured from 3D scanning, e.g., CT, or utilizing a one-size-fits-all standard model. However, considering the limited availability of 3D imaging for dental surgeries, OralViewer generates the detailed oral cavity model from a single 2D panoramic X-ray with a novel 3D reconstruction algorithm. Second, no existing study enables the 3D demonstration of dental surgeries, which we explore in the design and implementation of OralViewer.

\subsection{Computer-Aided Design (CAD) for Dentistry}

CAD tools have been widely applied in dentistry to improve the design of dental restorations, e.g. crowns, dental implants and orthodontic appliances [14, 39]. Specifically, models of patients' oral cavity are created from digital 3D scanning, based on which dentists produce a virtual design of restorations for manufacturing [34]. However, all the CAD tools are aimed to guide a clinician through restoration designing $[45,46]$, rather than patient education, which is the focus of OralViewer. Thus, oral cavity visualization and surgical step simulation have not been considered in existing CAD tools when it comes to patient education. Moreover, 3D imaging of patient's oral cavity, e.g., CT and intra-oral scanning [14,38], is almost always required by the existing CAD tools. In contrast, OralViewer reconstructs the patient's $3 \mathrm{D}$ oral cavity from the $2 \mathrm{D}$ panoramic $\mathrm{X}$-ray, which is one of the most common imaging modalities in dentistry [50], in order to enable the application of the system for a wide range of dental surgeries.

\subsection{Single-View 3D Reconstruction}

Single-view 3D reconstruction aims at generating the 3D model of an object based on a single 2D projection of it. Currently, deep Convolutional Neural Networks (ConvNets) have achieved the highest accuracy in various benchmarks by using both low-level image cues, e.g., texture, and high-level semantic information [47, 53]. Our work targets at generating the voxel-based representation of teeth volumes, which estimates a voxel occupancy grid for indicating if voxels are within the space of an object. The representation selection mainly considers the need for smooth and closed-surface models, even with the presence of complex typologies on occluded surfaces. A few existing work $[1,2]$ explored single tooth reconstruction from radiology; [27] initialized the teeth reconstruction with synthesized images; and [43] developed the back-projection of Cone Beam CT from X-ray. However, all those works cannot serve our purpose of patient-specific comprehensive dental structure modeling from clinical 2D panoramic X-rays.

\section{FORMATIVE STUDY}

To understand the system requirements of OralViewer from a clinical point of view, we conducted interviews with three dentists (two female and one male). We started by asking for the method he/she applies to perform patient education. We then described the motivation and goal of OralViewer, emphasizing on using a 3D model to visualize and simulate surgical steps to laymen patients. We gathered and built on dentists' feedback to formulate the below system requirements that ensure clinically-valid demonstration and user-friendliness.

R1. Providing patient-specific teeth model. Digital models are more favorable than physical models (e.g. gypsum models), since physical ones are fix and cannot reflect the effect of a surgical steps. Comparing between general digital models and patient-specific digital models, patient-specific ones are preferred by dentists to explain why a certain surgical step is carried out or in a specific way. Specifically, the decision of surgical steps, e.g. how a fractured tooth is extracted or repaired, often depend on individual's teeth condition. Thus, patient-specific teeth model should be provided to make demonstrations contextualized without misleading patients by inconsistent conditions. Moreover, compared to panoramic oral $\mathrm{X}$-ray, 3D screening of oral cavity is not a standard practice for the clinical diagnosis of many common surgeries, e.g. apicoectomy, root canal treatment, and crown lengthening, for its higher radiation and cost. As such, it is preferred to generate a patient's 3D teeth model from his/her 2D X-ray image to enable the widely available application of the system.

R2. Modeling complete oral cavity. Both the target oral structure of a surgery and its nearby anatomies need to be incorporated into a surgical demonstration. For example, when dentist removes a root tip in apicoectomy, procedures on other structures should be simulated as well, e.g. some gum tissue will be lifted from an area near the root tip and some surrounding bone will be removed. Thus, to help patients understand what to expect in a surgery, complete oral cavity including teeth, gum, and jaw bones should be modeled.

R3. Demonstration in simple operations. Dentists consider it important to show for each surgery step: ( $i$ ) how the step is performed - illustrating the applied instruments, and ( $i i)$ what happens in the step - animating the dental structure changes upon the application of instruments. Moreover, the demonstration should be carried out by dentists using simple interaction techniques, which is more important than having to achieve realistic effects with a high fidelity. For example, to demonstrate shaping a tooth with a dental handpiece, dentists prefer a simple operation, e.g., pressing and dragging the cursor on desired places of a tooth with customizable effect to simulatively perform a grinding as in an actual surgery. 


\section{ORALVIEWER DESIGN AND IMPLEMENTATION}

Guided by the aforementioned requirements, we designed and implemented OralViewer for 3D demonstration of dental surgery to patients. The OralViewer consists of two cascaded parts: (i) a 3D reconstruction pipeline for generating a patient's oral cavity from a single 2D panoramic X-ray, and (ii) a demonstration tool for dentist's animating steps on the 3D model with virtual dental instruments.

\subsection{D Reconstruction of Oral Cavity}

OralViewer reconstructs a complete oral cavity model consisting of teeth, gum and jaw bones, all of which are vital for the demonstration of surgical procedures (R2). Importantly, to reflect the patient-specific dental condition, we estimate the patient's 3D teeth structures from a single 2D panoramic X-ray with an end-to-end trainable deep ConvNet model (R1). Since the 3D structures of soft tissues, i.e., gum, and jaw bones cannot be well reflected from X-ray [19], their 3D templates are pre-defined and can be registered to tailor for specific patients' oral anatomy.

Model Architecture. Our task of teeth reconstruction has two unique challenges from the existing voxel-based work. (i) The reconstruction contains multiple objects (teeth) rather than a single object as in $[13,47,53]$. (ii) The input image of X-ray has a higher resolution than existing work (e.g., $128 \times 128$ [13]), which calls for higher computational and memory efficiency of model. To tackle both challenges, we decompose the task into two sub-tasks of teeth localization and patch-wise single tooth reconstruction. Figure 2 shows the overall architecture of our model. The input 2D panoramic X-ray $X \in \mathbb{R}^{H \times W}$ (Figure 2(a)), where $H$ and $W$ are image height and width, is fed to a feature extraction subnet (Figure 2(a)) of a 2D encoder-decoder structure for capturing a deep feature map of the same resolution as the input X-ray. Given the feature map, a segmentation subnet (Figure 2(b)) formulates an anatomical segmentation task $[25,26]$ to map it into a categorical mask $Y_{\text {seg }} \in \mathbb{Z}^{H \times W \times K}$, where $K=32$ denotes the maximum number of tooth category. Figure 4 a demonstrates the tooth numbering rule we used by following the World Dental Federation Notion [20]. Moreover, the tooth localization of bounding boxes is further derived from the segmentation map by keeping the largest island per tooth, as shown in Figure 2(3). According to the tooth localization, a tooth reconstruction subnet (Figure 2(c)) performs patch-sampling for all teeth from the aforementioned deep feature map (Figure 2(2)), and back projects them into 3D tooth shapes represented in 3D occupancy map (Figure 2(4)), using a 2D-encoder-3D-decoder structure similar to [13]. Note that the deep feature map (Figure 2(2)) is shared for both segmentation and tooth reconstruction sub-tasks in order to increase the compactness and generalization of the model. By assembling the predicted tooth volumes (Figure 2(4)) according to their estimated localization from X-ray segmentation (Figure 2(3)), we can achieve a flatten 3D reconstruction of teeth, which is then bent to an estimated dental arch curve (Figure 2(5)) for the final 3D reconstruction as shown in Figure 2(6)). The parameters in all the subnets of the model (Figure $2(a, b, c)$ ) can be optimized in an end-to-end fashion for the optimal performance. More details about the model can be found in the Appendix.

Unsupervised Dental Arch Curve Estimation. The dental arch curve needs to be estimated since such information is lost during the rotational screening process of a panoramic X-ray imaging system [19]. Multiple methods can be applied for the estimation, e.g., using average shape from general population [32], and $\beta$-curve fitting with measured width and depth of oral cavity [9]. In this work, we propose a semi-automatic pipeline to accurately extract dental arch curve from occlusal surface photos without supervision, as shown in Figure 3. First, macro shots of upper and lower occlusal surfaces are taken from a patient (Figure 3(a)), and are roughly labeled for teeth regions with simple sketching (Figure 3(b). The dental arch area can then be obtained with the active contour algorithm [52] applied on the sketches as priors (Figure 3(c)), and further skeletonlized for an initial dental arch curve (Figure 3(d)). The final smooth dental arch curve is achieved by fitting a cubic curve to the uniformly sampled data points from the initial curve (Figure 3(e)).

Complete Oral Cavity Model. To achieve a complete oral cavity model, OralViewer is embedded with a set of pre-built template models for gums and jaw bones. As shown in Figure 4b, to build the templates, a Cone Beam CT from an adult male was collected $4 \mathrm{~b}(\mathrm{a})$, and pre-processed with intensity thresholding [33] for extracting the skull structure $4 \mathrm{~b}(\mathrm{~b})$. Then, the upper jaw (Figure $4 \mathrm{~b}(\mathrm{c} 1)$ ) and lower jaw (Figure $4 \mathrm{~b}(\mathrm{~d} 1)$ ) bone models were constructed by removing tooth structures, hole filling, and smoothing, while the upper gum (Figure $4 \mathrm{~b}(\mathrm{e} 1)$ ) and low gum (Figure $4 \mathrm{~b}(\mathrm{f} 1)$ ) models were constructed as the smooth volumes embodying the corresponding jaw bones. For the reconstruction for each patient in the deployment stage, the pre-built gum and jaw bone models are first registered and aligned to the estimated dental arch curves (Figure $4 \mathrm{~b}(\mathrm{~g})$ ). Then the deformed gum and jaw bones model (Figure $4 \mathrm{~b}(\mathrm{c} 2, \mathrm{~d} 2, \mathrm{e} 2, \mathrm{f} 2)$ ) are assembled with the $3 \mathrm{D}$ reconstructed teeth for the complete oral cavity model as the example shown in Figure $4 \mathrm{~b}(\mathrm{i})$. We expect the averaging models of gum and jaw bone from CT scans of multiple individuals can further improve the reconstruction quality, while the current templates have been shown valid for the patient education purpose according to dentists as detailed in the Expert Study section.

Training Dataset. OralViewer utilizes a deep ConvNet for estimating patient-specific 3D teeth structure from a 2D panoramic $\mathrm{X}$-ray. Intuitively, the model can be trained with the supervision of patients' paired data of 3D teeth structures obtained from the teeth labeling of CT and panoramic X-ray. However, tooth structures from X-ray and CT are misaligned due to different postures during screening, e.g. head directions and occlusion condition. As such, we propose to collect CT scans, and synthesize their corresponding panoramic X-rays as the model input. The synthesis is valid since the CT scans contain full 3D information of oral cavity, while panoramic radiographs are the $2 \mathrm{D}$ projections of them. Several previous work has demonstrated promising results for high quality $\mathrm{X}$-ray synthesis from CT, and we employed the method from Yun et al. [30] in this work. In total, our in-house dataset contains 23 pairs of 3D CT scans and synthesized panoramic X-rays. Moreover, 


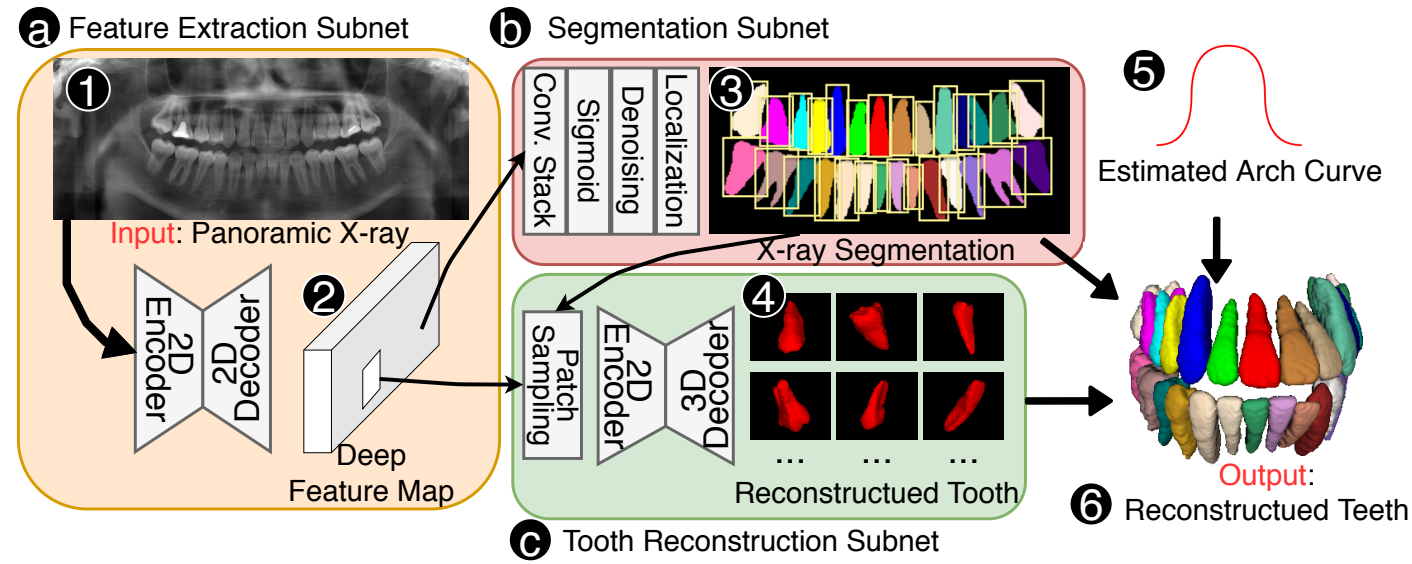

Figure 2: Overall model architecture for reconstructing 3D tooth structures from a panoramic X-ray.

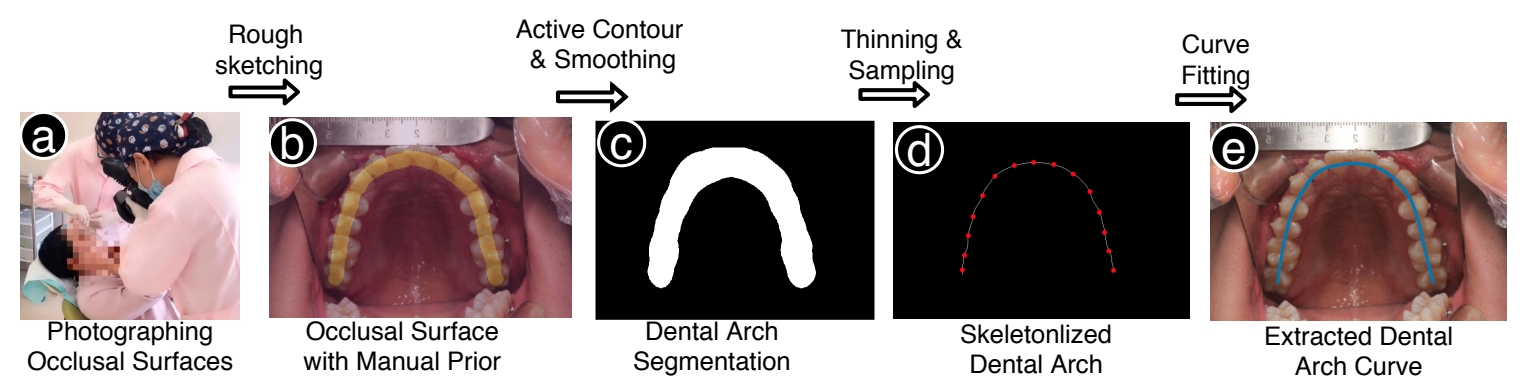

Figure 3: Semi-automatic dental arch curve estimation from occlusion photos.

a

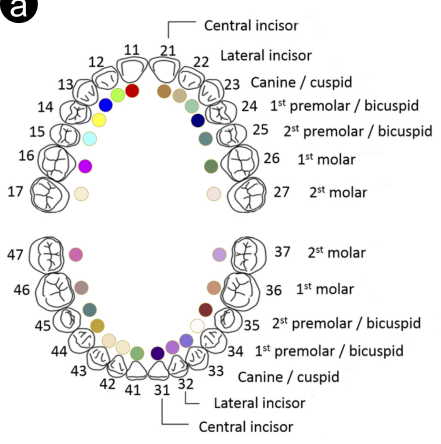

b

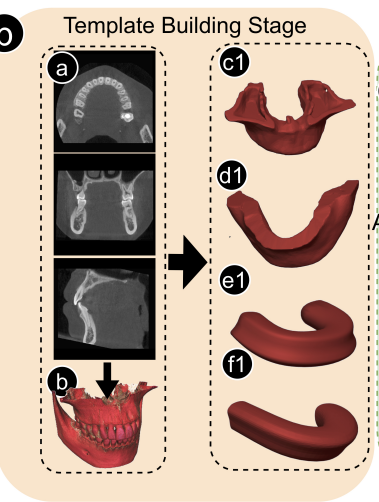

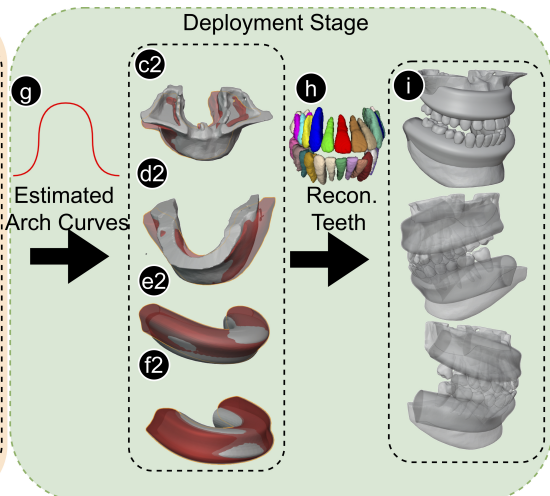

Figure 4: (a) Teeth naming, numbering and coloring rule. (b) Gum and jaw bone templates building method, and their application in the deployment stage for a complete oral cavity model.

another 39 real panoramic X-ray scans have also been included for augmenting the training process of the segmentation subnet of the model. All CBCT scans and panoramic X-rays have been first labeled with pixel-wise tooth masks by 3 research team members, and then reviewed by 2 board-certificated dentists. We randomly split the paired data into 21 scans for training, and 2 scans as the validation set for determining the early stopping of the training process.
Training Strategy. We employed a two-stage training paradigm. In the first stage, we train the deep feature extraction subnet and segmentation subnet (Figure 2(a,b)) for tooth localization. For the segmentation loss $L_{\text {seg, }}$, we define it to be the average of dice loss across all tooth categories. In the second stage, we train the whole model including tooth reconstruction subnet (Figure 2(c)) for the optimal performance of both tooth localization and reconstruction, with the loss defined as the sum of the aforementioned 
segmentation loss $L_{\text {seg }}$ and a reconstruction loss $L_{\text {recon }}$. The $L_{\text {recon }}$ was set as the $3 \mathrm{D}$ dice loss between the ground-truth volume and the predicted volume. More details about model training can be found in Appendix.

\subsection{Demonstrating Surgery with Virtual Dental Instruments}

OralViewer provides a web-based tool for dentists to demonstrate surgery steps on a patient's oral cavity model with virtual dental instruments. The dental instruments allow dentists to express what and where an action is applied to the oral cavity, and demonstrate the effect on the model in real-time (R3). Moreover, dentists can use simple sliders to customize the animation effect of the instruments to better suit their particular needs and preferences. By discussing with the dentists, the current tool's implementation consists of six common dental instruments : (1) surgical scalpel, (2) fissure bur, (3) handpiece, (4) syringe, (5) curette, and (6) artifacts. In this section, we first show the overall workflow of using the tool, and then describe the technical details of each virtual dental instrument, followed by the tool implementation.

Overall Workflow. As shown in Figure 5(a), a dentist start by importing a reconstructed 3D oral cavity model generated from the aforementioned pipeline (Figure 5(1)), which can be viewed freely with rotation and scaling. To apply a virtual dental instrument, the dentist selects the instrument from a list (Figure 5(2)). Upon the selection, the corresponding instrument model (Figure 5(4,6)) is visualized, and can be controlled by using the mouse to move and operate on the oral cavity model. For instruments 1-5, e.g., scalpel as shown in Figure 5(b), their animation effect on dental structures can be customized by changing a set of parameters (Figure 5(3)); while for dental artifacts, e.g., implant as shown in Figure 5(c), their shapes and directions can also be adjusted to tailor for the patient's condition (Figure 5(5)). The selected instrument can be directly applied to a dental structure for demonstrating effects with clicking, pressing, and dragging (Figure 5(d)). The oral cavity model can also be freely rotated by press-and-drag and scaled with wheel to adjust an optimal view for the demonstration and manipulation. The effect can be dynamically reflected on the structure in real-time, with the operated structure highlighted for visualization (Figure 5(7)). A typical dental surgery consists of several sequential steps using multiple dental instruments, which can be demonstrated with each dental instrument incrementally by repeating the steps of instrument selection (Figure 5(a)), adjusting (Figure 5(b,d)), and animating (Figure 5(d)).

\#1 Surgical Scalpel \& \#2 Fissure Bur. Both tools are used to incise dental structures: surgical scalpel can be applied to gum tissue for creating periodontal flap; while fissure bur can create holes on jaw bones for the exposure of the root tip. To use the tools, a dentist describes the incision location and its size by creating a region from a sequence of mouse clicks, where the created boundary is visualized with red lines (Figure 6(b,f)). Upon a closed-loop boundary is formed, the incised region is highlighted (Figure 6(c,g)), and the corresponding structure is then removed upon the dentist's confirmation. The design allows dentists to perform any type of incision according to a patient's condition, e.g., semilunar and triangular types of periodontal flap. Figure $6(\mathrm{a} \rightarrow \mathrm{d})$ shows a periodontal flapping using surgical scalpel, and Figure $6(\mathrm{e} \rightarrow \mathrm{h})$ shows a jaw bone opening based on a flapped gum.

\#3 Handpiece. Handpiece is widely used for shaping bone structures, e.g., tooth and jaw bones. A dentist can move the virtual handpiece as mouse cursor to any desired location of grinding. The grinding effect takes place once with a mouse click, or continuously by pressing and dragging the mouse. The size and intensity of the grinding effect can be customized using sliders. For example, Figure $6(\mathrm{i} \rightarrow \mathrm{j})$ shows the resection of an exposed root tip in an apicoectomy; while Figure $6(\mathrm{k} \rightarrow \mathrm{l})$ demonstrates the reduction of jaw bone height in a crown lengthening surgery.

\#4 Syringe \& \#5 Curette. Both instruments can be used to fill materials into bone openings. A dentist can move either instrument as a cursor to the desired location on a tooth or jaw bone, and click or press-and-drag for the filling effect. The size and intensity of the filling effect can be customized using sliders. For example, Figure $7(\mathrm{a} \rightarrow \mathrm{b})$ shows the syringe is used to inject bone grafting materials to fill the jaw bone opening, and Figure $7(\mathrm{c} \rightarrow \mathrm{d})$ demonstrates a curette is applied on a fractured crown for restoration.

\#6 Artifacts. Artifact crown and implant are included in the demonstration tools. To apply them, a dentist starts with importing a pre-defined artifact model, followed by specifying the dental structure to be applied on. The artifact model and the dental structure are visualized in blue and red (Figure $7(\mathrm{e}, \mathrm{g})$ ) for assisting the operations and demonstration. The 3D location of the artifact can be adapted with dragging in $2 \mathrm{D}$ viewing planes, while both the orientation and size of the artifact can be modified to match the patient's condition using sliders from $X, Y$, and $Z$ axis. Once the artifact has been customized with confirmation from dentists, the artifact model and the operated dental structure are merged as one object. Figure $7(\mathrm{e} \rightarrow \mathrm{f}, \mathrm{g} \rightarrow \mathrm{h}$ ) shows the cementation of an implant on a resected root and a dental crown on a prepared tooth, respectively, as indicated with red arrows.

Implementation. The demonstration tool of OralViewer was implemented using OpenGL, JavaScript and three.js. The tool can run readily inside a modern browser. The effect of gum/jaw bone incision with surgical scalpel/fissure bur and artifact implanting was implemented with the Constructive Solid Geometry (CSG) operations ${ }^{1}$ between: (i) generated 3D convex geometry from the input trajectories or pre-built artifact models, and (ii) corresponding dental structures. The effect of shaping/filling with handpiece/syringe/curette was implemented with mesh sculpting operations ${ }^{2}$ including flattening, filling, and scraping.

\section{EVALUATION}

We conducted $(i)$ a technical evaluation for reconstructing a 3D model of a patient's oral cavity, (ii) a patient study with 12 participants, and (iii) a preliminary expert study with three board-certified dentists.

\footnotetext{
${ }^{1}$ https://github.com/oathihs/ThreeCSG

${ }^{2}$ https://stephaneginier.com/sculptgl/
} 


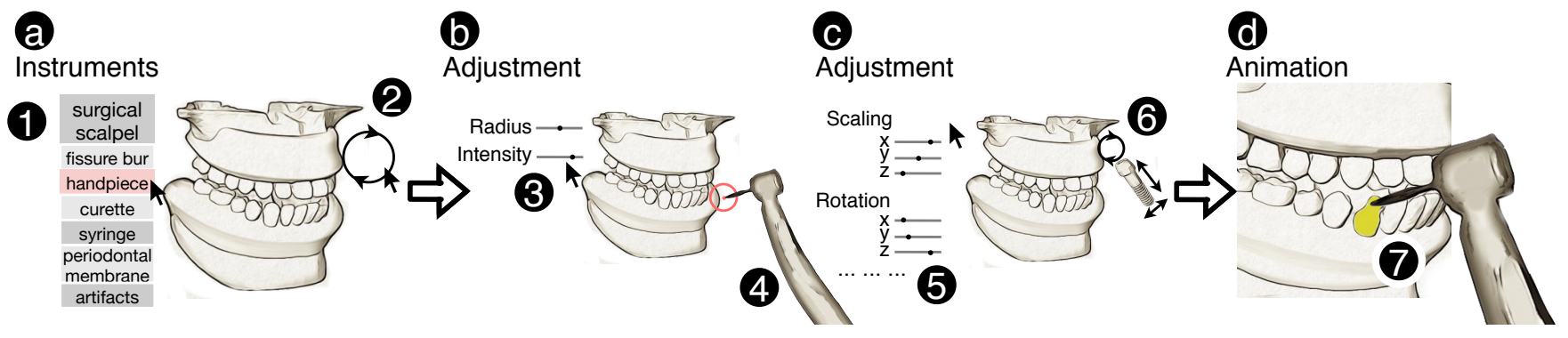

Figure 5: Overall workflow for demonstrating dental surgeries.
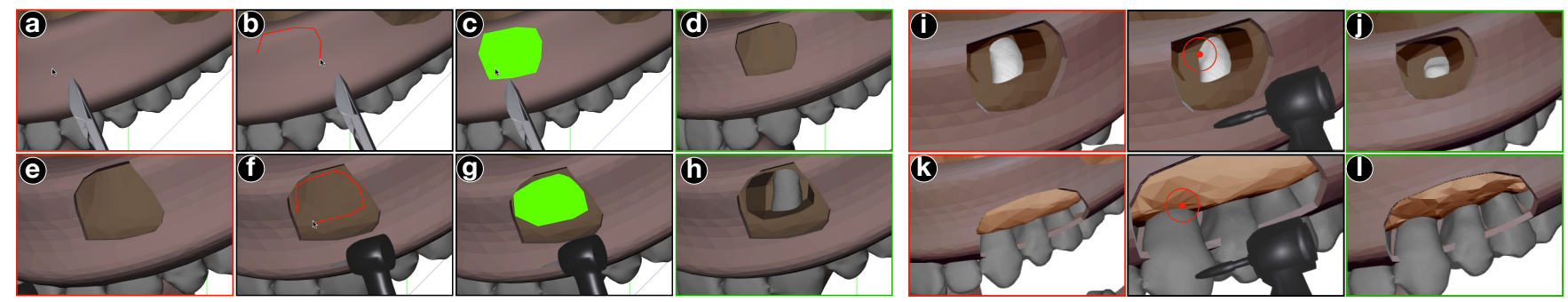

Figure 6: Example use cases of virtual surgical scalpel (top left), fissure bur (bottom left), handpiece for tooth tip resection (top right) and jaw bone lowering (bottom right).
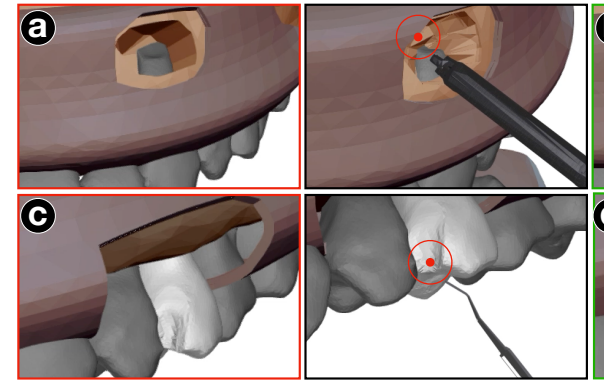
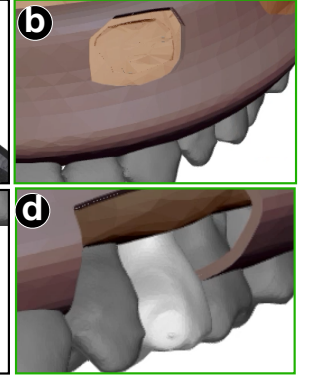
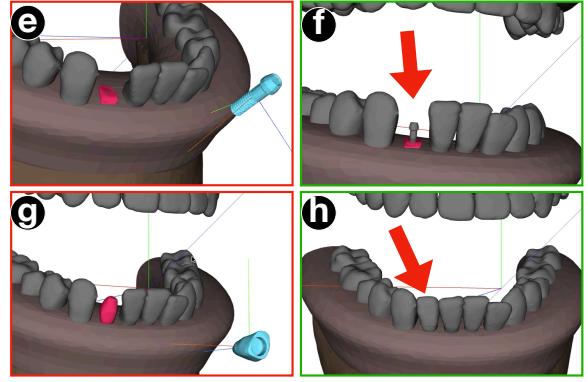

Figure 7: Example use cases of syringe for injecting grafting materials (top left), curette for restoring fractured crown (bottom left), applying an artifact implant (top right), and cementing an artifact crown (bottom right).

\subsection{Technical Evaluation}

Dataset. We built an in-house testing dataset collected from 10 patients from a local orthodontics hospital. Each patient was screened for both panoramic X-ray and Cone Beam CT. Moreover, since our reconstruction pipeline estimates patient-specific dental arch curves, two photos of occlusion macro photos (for upper and low jaws respectively) of each patient were captured by dentists in the clinic. To quantitatively evaluate the reconstruction accuracy, patients' tooth structures were manually labeled from CT scans by two research team members and reviewed by one dentist.

Results \& Analysis. We applied our reconstruction pipeline with the panoramic X-ray and occlusion photos as input to generate the complete $3 \mathrm{D}$ oral cavity model. We first evaluate the teeth reconstruction accuracy for reflecting patient-specific condition by quantitatively comparing the generated model with labeled 3D structure from CT. We used the Intersection over Union (IoU) between the predicted volume $P$ and the ground-truth volume $G$ of each tooth as the performance metric, where $I o U=|P \cap G| /(|P \cup G|)$, and $|$.$| denotes the cardinality of a voxel set. To overcome the$ misalignment of teeth between X-ray and CT caused by patients' gestures, the upper and lower teeth labeling from CT (3D) was rigidly aligned (using ANTs [6]) to the corresponding part of the reconstructed 3D teeth (3D) from the model before the IoU calculation. Figure 8 (a) reveals the IoUs for all the 32 categories of tooth, where our model achieves a mean IoU of 0.771 , with patient-wise $s t d=0.031$ and tooth category-wise $s t d=0.062$. The result indicates the effectiveness of our method for reconstructing 3D teeth model from 2D x-ray image. Yet, we also find that the method has a consistent lower accuracy for wisdom teeth (numbering 18, 28, 38 , and 48) than the others, which can be caused by: (i) the shape of wisdom teeth is more variant across different persons, and (ii) relatively fewer samples are available for ConvNet training (16 out 
of 23 cases in the training dataset containing one or more wisdom teeth).

We then qualitatively evaluate the 3D reconstruction of the complete oral cavity model. Figure 9 visualizes an example case from the testing dataset: the input panoramic X-ray (a), the semi-automatic dental arch extraction results for both upper and lower jaws (b), the reconstructed 3D teeth model from the $2 \mathrm{D} \mathrm{X}$-ray and the estimated dental arch curves (c), the ground-truth teeth structures extracted from the CT image (d), and the complete oral cavity model with the pre-defined jaw bone and gum models registered and assembled with the reconstructed teeth (e). Note that the shown case has 24 teeth, rather than 28 teeth as a normal adult. This is possibly because 4 teeth have been extracted during a previous orthodontic operation. The reconstructed results clearly reflect this individual's condition, and show the effectiveness of our reconstruction pipeline for generating $3 \mathrm{D}$ oral cavity models.

\subsection{Patient Study}

OralViewer enables a dentist to help laymen patients understand procedures of dental surgeries by $3 \mathrm{D}$ demonstration. To validate the feasibility of our approach, we investigate the research question concerning the effect of OralViewer:

- RQ1: can OralViewer improve patients' understanding of a surgery?

6.2.1 Participants. We recruited 12 patients from the clinic of an orthodontics hospital (4 females and 8 males, aged between 21 to 64 years). Each participant was demonstrated with one surgery of crown lengthening or apicoectomy. None of the participants had received the dental surgery before. Note that due to limited patient resources, we were only able to recruit participants who came in for regular dental check-up but did not actually undergo such dental surgeries. The detailed demographic information of our participants can be found in Appendix.

6.2.2 Procedure. The patient study consists of the following key activities:

Tutoring dentists how to use the system. To clinically validate OralViewer, we collaborated with three board-certified dentists (E1: male, 25 years of practice; E2: male, 17 years of practice, E3: female, 11 years of practice) for carrying out the surgery demonstrations. We first introduced OralViewer, let each dentist follow a step-by-step onboarding tutorial, and answered their questions about utilizing the program. Then dentists were free to continue trying out OralViewer's virtual dental instruments until they felt they were able to use the system independently.

In-clinic study. In order to compare OralViewer with current patient education method, we randomly split the participants into an experiment group of 7, which was demonstrated OralViewer, and a control group of 5, which was demonstrated an X-ray and verbal descriptions as per the dentists' regular practice. Participants were randomly assigned one of the two dental surgeries, e.g., apicoectomy or crown lengthening, to receive a demonstration: 4 participants in the experiment group and 3 in the control group were demonstrated apicoectomy; while 3 in the experiment group and 2 in the control group were demonstrated for crown lengthening. Each study happened in one of the three dentists' clinics and
OralViewer was accessed as a web app using dentists' own computers (Figure 8(b)) . Details on the surgery and dentist assignment can be found in Appendix.

Exit interview. After the explanation, we interviewed each participant to verbally describe the surgery procedures by focusing on: ( $i$ ) what steps are included, and (ii) how (by using what instrument) a procedure is applied. Their answers were recorded and later compiled for evaluating their understanding of surgeries.

6.2.3 Analysis \& Results. We scored the participants' understanding on a surgery based on whether the key steps of the surgery were described in their answers. Specifically, there are five key steps for apicoectomy: (S1) periodontal flap, (S2) jaw bone opening, (S3) root tip removal, (S4) root tip sealing, and (S5) grafting material injection; while five key procedures were considered for crown lengthening: (S1) periodontal flap, (S2) jaw bone shaping, (S3) tooth preparing, and (S4) artifact implanting. An answer regarding a step is scored as: 0 if the step was not described, 1 if the step was described but the applied dental instrument was not, and 2 if both the step and its dental instrument were mentioned or described. Note that the exact names of step/instrument were not required to be mentioned in an answer for a score - a step/instrument was counted if it was described or indicated from a patient's answer. Figure 10 shows the average score of each step within the experiment and control group for the apicoectomy (Figure 10(a)) and crown lengthening (Figure 10(c)). We can see that OralViewer significantly improves patients' understanding in three out of the five steps for apicoectomy and two out of the four steps in crown lengthening, while the improvement in the other steps is not statistically significant. Moreover, the overall average score among all steps between the experiment and control group was 1.36 vs. 0.85 , indicating OralViewer can improve patients' understanding significantly $(p<0.05)$.

\subsection{Preliminary Expert Study}

To clinically validate OralViewer, we interviewed 3 dentists from the patient study after they finished all the assigned patient demonstrations. Each dentist had done at least one demonstration using OralViewer (E1: 3 times; E2: 2 times; E3 2 times. Details in Appendix). We investigate the following questions:

- RQ2: Usability - do dentists have difficulty using virtual dental instruments in OralViewer?

- RQ3: Validity - is OralViewer's demonstration effect clinically valid for patient education?

- RQ4: Preference - do dentists prefer a system like OralViewer as a tool for performing patient education for surgeries?

We asked each dentist to rate their agreement (from 1-strongly disagree to 7-strongly agree) with statements about instruments' usability and demonstration effect, and the demonstration effect of 3D oral cavity models (RQ2 \& 3), as well as their preference (RQ4).

6.3.1 Usability. We measured the usability for the four types of virtual instruments involved in the demonstration of apicoectomy and crown lengthening: (S1) surgical scalpel and fissure bur for gum/jaw incision, (S2) handpiece for jaw/tooth shaping, (S3) syringe and curette for tooth filling/material injection, and (S4) dental artifacts for implanting. Figure 11(a) shows the questions and experts' scores. While all the experts successfully carried out all the 

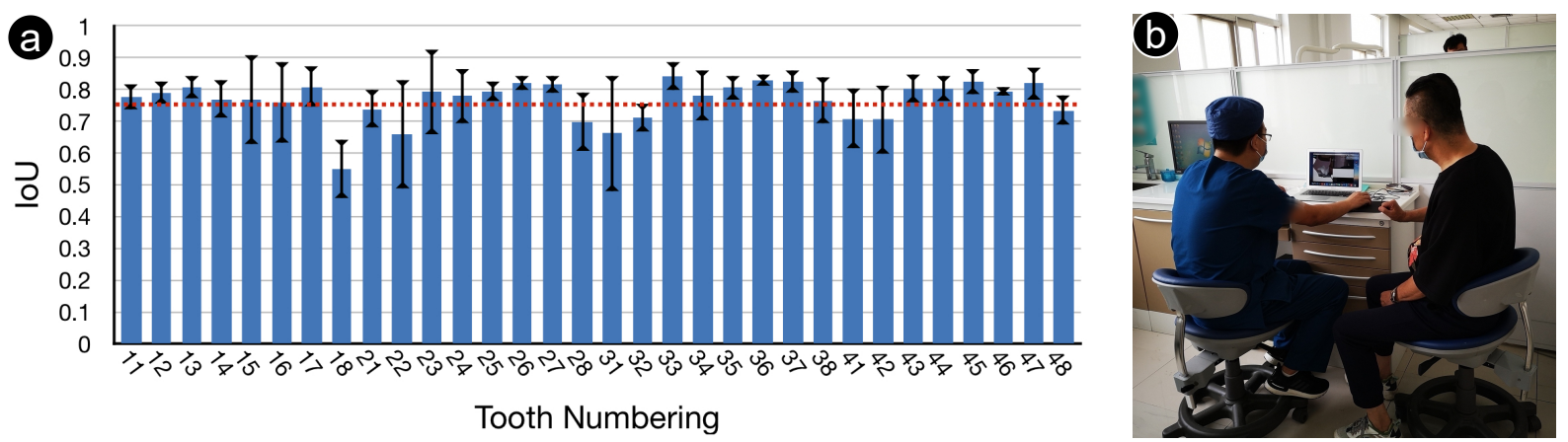

Figure 8: (a): 3D teeth reconstruction accuracy. (b) A participant was demonstrated a dental surgery using OralViewer by a dentist.
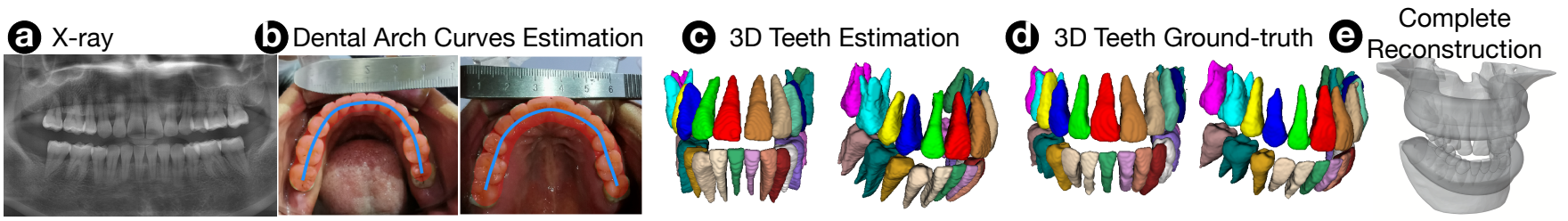

Figure 9: Reconstruction example from testing dataset.
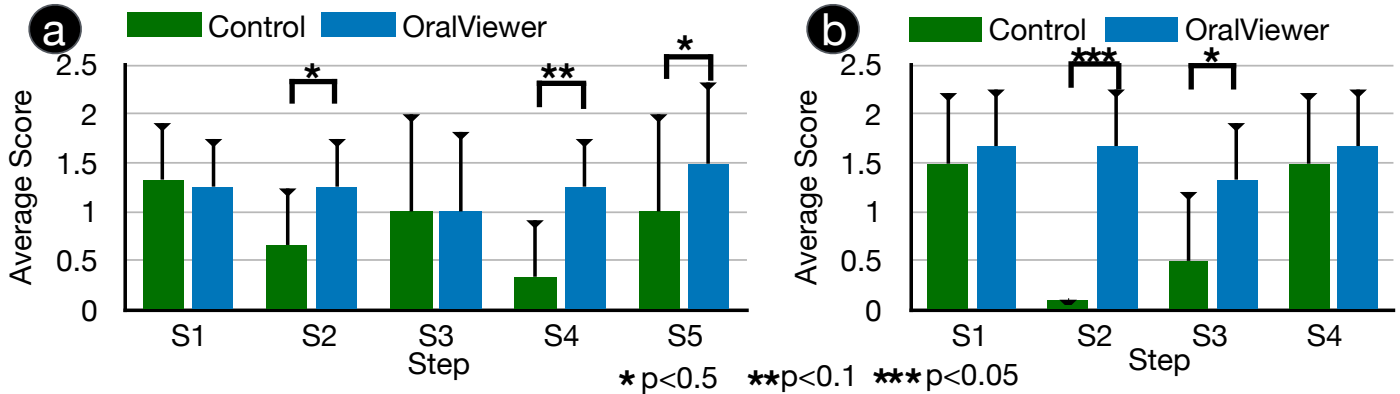

Figure 10: Participants' understanding scores for (a) apicoectomy and (b) crown lengthening. Sx represents key steps.

demonstrations with patients using OralViewer, a major issue raised by experts was that the virtual instrument control with mouse was unfamiliar to dentists: it is different from the way dentists using real dental instruments in a surgery, which can lead to a steep learning curve (E2,E3). E3 suggested that an implementation of OralViewer on touch screen, e.g., iPad, with the control of virtual instruments using stylus should be more intuitive to dentists.

6.3.2 Validity. The experts rated the demonstration effect of OralViewer for the 3D oral cavity model (S1) and the four types of virtual instruments (S2-S5). Figure 11(b) shows the questions and experts' ratings, where OralViewer achieves a mean score of 5.67 out of 7 . All experts agreed that our reconstructed oral cavity model and virtual instruments are clinically valid for patient education. Regarding the oral cavity model, experts confirmed that it contributes to the surgical demonstration because $(i)$ patients are able to see structures that they cannot observe from a mirror, e.g., molar teeth, using rotation in 3D (E1, E2, E3); and (ii) the patient-specific teeth can not only let patients understand their conditions better (E2, E3) but also raise their interests in learning more about operating on such conditions (E3). Besides, E3 suggested that oral cavity model can be improved by modeling root canals within tooth (detail in the Discussion section). Regarding virtual instruments, experts agreed that they are valid for the patient education purpose (E1, E2, E3), and preferred the visualization of the instruments, which can help patients comprehensively understand what to expect during a surgery (E3). Moreover, experts also suggested that the appearance of virtual instruments can be dynamic altered upon users customizing their effect to further improve the visualization (detailed in the Discussion section).

6.3.3 Preference. We asked experts about their preference of OralViewer from two perspectives: (S1) OralViewer enables effective patient education and (S2) I would integrate OralViewer into my existing practice. As shown in Figure 11(c), the experts agreed that OralViewer enables effective patient education with a mean score 


\begin{tabular}{|} 
a 1: Strongly disagree -7: Strongly agree \\
\hline E1 & E2 & E3 & Mean \\
\hline (S1) I had no trouble using virtual scalpel/fissure bur. \\
\hline 4 & 5 & 3 & 4.00 \\
\hline (S2) I had no trouble using virtual handpiece. \\
\hline 4 & 4 & 5 & 4.33 \\
\hline (S3) I had no trouble using syringe/curette. \\
\hline 3 & 4 & 5 & 4.00 \\
\hline (S4) I had no trouble using virtual artifacts. \\
\hline 3 & 5 & 5 & 4.33 \\
\hline C C & 5 & 6.00 \\
\hline (S1) OralViewer enables effective patient education. \\
\hline 5 & 7 & 6 & 5.00 \\
\hline (S2) I would integrate OralViewer into my work pipeline. \\
\hline 5 & 6 & 4 & 6 \\
\hline
\end{tabular}

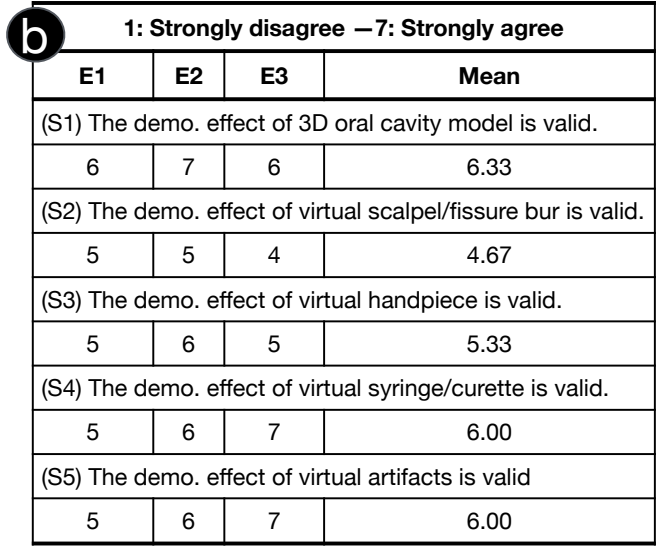

Figure 11: Experts' scores for OralViewer about (a) usability, (b) demonstration effect validity, and (c) preference.

of 6.00 out of 7 . The experts also rated agreement of 5.00 out of 7 for integrating OralViewer into their existing practices. As mentioned by E2, the tool can be very necessary with the patients' recently growing need for improved dentist visit experience and their willingness to involve in treatment planning. He also pointed out that animating procedures on patient-specific model can possibly contribute to higher patient satisfaction because of the personalized communications.

\section{LIMITATIONS, DISCUSSIONS, AND FUTURE WORK}

\subsection{Improving Oral Cavity Modeling}

We enable 3D oral cavity modeling by assembling patient-specific teeth structures estimated from X-ray and registered gum and jaw bone templates. However, two improvements can be made according to expert interviews. First, the current gum templates as the smooth volumes embodying a set of CT-extracted jaw bones are reported to be coarse in form (E3). Although sufficient for patient education, E3 suggested that improved gum templates should be pre-built from existing intra-oral scans that are capable of capturing soft tissue. Second, the current reconstructed teeth do not model root canals, which can be useful in certain surgeries, e.g., root canal treatment (E3). Future work can enable the root canal modeling by either augmenting the current solid teeth model with artifact canals or including the root canal modeling in the ConvNet training process.

\subsection{Extending Virtual Instrument Set for All Dental Surgeries}

The current implementation of OralViewer includes 6 common dental instruments. However, more virtual instruments are required towards a comprehensive system for all dental surgeries, e.g., endodontic files for root canal treatment. Since most virtual instrument effect can be simulated with CSG (Constructive Solid Geometry) operations, e.g., Boolean operation between two models, and mesh sculpting, e.g., smoothing, creasing, and flattening, we suggest that OralViewer should be extended to allow dentists to register new virtual dental instruments. Specifically, various CSG and mesh sculpting functions can be implemented and a dentist can add a new instrument by importing a instrument model for visualization and its parameters for configuring the operating effects.

\subsection{Improving Dental Instrument Visualization}

OralViewer visualizes dental instrument for enhancing patients' understanding. However, expert E3 suggested that the visualization should be improved from two aspects. First, when the animation effect of the instrument is changed, the appearance of the instrument can be dynamically altered to reflect the effect change. For example, the head of a handpiece can become larger when the effecting size of grinding is set to be larger. Second, different models of each type of instrument can be pre-defined for dentists' selection to reflect the real surgery situation.

\section{REFERENCES}

[1] Aly S Abdelrahim, Moumen T El-Melegy, and Aly A Farag. 2012. Realistic 3d reconstruction of the human teeth using shape from shading with shape priors. In 2012 IEEE Computer Society Conference on Computer Vision and Pattern Recognition Workshops. IEEE, 64-69.

[2] Aly S Abdelrehim, Aly A Farag, Ahmed M Shalaby, and Moumen T El-Melegy. 2013. 2D-PCA shape models: Application to 3D reconstruction of the human teeth from a single image. In International MICCAI Workshop on Medical Computer Vision. Springer, 44-52.

[3] Stewart Agras, David Sylvester, and Donald Oliveau. 1969. The epidemiology of common fears and phobia. Comprehensive psychiatry 10, 2 (1969), 151-156.

[4] Jason M Armfield and LJ Heaton. 2013. Management of fear and anxiety in the dental clinic: a review. Australian dental journal 58, 4 (2013), 390-407.

[5] Koula Asimakopoulou, Adyya Gupta, and Sasha Scambler. 2014. Patient-centred care: barriers and opportunities in the dental surgery. Community dentistry and oral epidemiology 42, 6 (2014), 603-610.

[6] Brian B Avants, Nick Tustison, and Gang Song. 2009. Advanced normalization tools (ANTS). Insight j 2, 365 (2009), 1-35.

[7] Ido Badash, Karen Burtt, Carlos A Solorzano, and Joseph N Carey. 2016. Innovations in surgery simulation: a review of past, current and future techniques. Annals of translational medicine 4, 23 (2016).

[8] Laila Bailey. 2010. Strategies for decreasing patient anxiety in the perioperative setting. AORN journal 92, 4 (2010), 445-460. 
[9] Stanley Braun, William P Hnat, Dana E Fender, and Harry L Legan. 1998. The form of the human dental arch. The Angle Orthodontist 68, 1 (1998), 29-36.

[10] Sophia Burghardt, Susan Koranyi, Gabriel Magnucki, Bernhard Strauss, and Jenny Rosendahl. 2018. Non-pharmacological interventions for reducing mental distress in patients undergoing dental procedures: Systematic review and meta-analysis. Journal of Dentistry 69 (2018), 22-31.

[11] Francesco Capuano, Yue-Hin Loke, Ileen Cronin, Laura J Olivieri, and Elias Balaras. 2019. Computational study of pulmonary flow patterns after repair of transposition of great arteries. Journal of biomechanical engineering 141, 5 (2019).

[12] Sung-Hwan Choi, Ji-Hoon Won, Jung-Yul Cha, and Chung-Ju Hwang. 2015. Effect of audiovisual treatment information on relieving anxiety in patients undergoing impacted mandibular third molar removal. Journal of Oral and Maxillofacial Surgery 73, 11 (2015), 2087-2092.

[13] Christopher B Choy, Danfei Xu, JunYoung Gwak, Kevin Chen, and Silvio Savarese 2016. 3d-r2n2: A unified approach for single and multi-view $3 \mathrm{~d}$ object reconstruction. In European conference on computer vision. Springer, 628-644.

[14] Gary Davidowitz and Philip G Kotick. 2011. The use of CAD/CAM in dentistry. Dental Clinics 55, 3 (2011), 559-570.

[15] John R Elter, RONALD P STRAUSS, and JAMES D BECK. 1997. Assessing dental anxiety, dental care use and oral status in older adults. The fournal of the American Dental Association 128, 5 (1997), 591-597.

[16] Kazuhiro Endo, Naohiro Sata, Yasunao Ishiguro, Atsushi Miki, Hideki Sasanuma Yasunaru Sakuma, Atsushi Shimizu, Masanobu Hyodo, Alan Lefor, and Yoshikazu Yasuda. 2014. A patient-specific surgical simulator using preoperative imaging data: an interactive simulator using a three-dimensional tactile mouse. fournal of Computational Surgery 1, 1 (2014), 1-8.

[17] Jörg Eschweiler, Jan-Philipp Stromps, Maximilian Fischer, Fabian Schick, Björn Rath, Norbert Pallua, and Klaus Radermacher. 2016. A biomechanical model of the wrist joint for patient-specific model guided surgical therapy: Part 2. Proceedings of the Institution of Mechanical Engineers, Part H: fournal of Engineering in Medicine 230, 4 (2016), 326-334.

[18] James L Gutmann and Paul E Lovdahl. 2010. Problem Solving in Endodontics-EBook: Prevention, Identification and Management. Elsevier Health Sciences.

[19] Joen Iannucci Haring and Laura Jansen. 2000. Dental radiography: principles and techniques. WB Saunders.

[20] Reinhard Hickel, Arnd Peschke, Martin Tyas, Ivar Mjör, Stephen Bayne, Mathilde Peters, Karl-Anton Hiller, Ross Randall, Guido Vanherle, and Siegward D Heintze. 2010. FDI World Dental Federation: clinical criteria for the evaluation of direct and indirect restorations-update and clinical examples. Clinical oral investigations 14, 4 (2010), 349-366.

[21] Steven Johnson, Kathleen Chapman, and Gene Huebner. 1984. Stress reduction prior to oral surgery. Anesthesia progress 31, 4 (1984), 165.

[22] Zeev N Kain, Ferne Sevarino, Gerianne M Alexander, Sharon Pincus, and Linda C Mayes. 2000. Preoperative anxiety and postoperative pain in women undergoing hysterectomy: A repeated-measures design. Journal of psychosomatic research 49 , 6 (2000), 417-422.

[23] Hakki Oguz Kazancioglu, Mustafa Tek, Seref Ezirganli, and Nihat Demirtas. 2015 Does watching a video on third molar surgery increase patients' anxiety level? Oral Surgery, Oral Medicine, Oral Pathology and Oral Radiology 119, 3 (2015), 272-277.

[24] Olaf E Langland, Robert P Langlais, and John W Preece. 2002. Principles of dental imaging. Lippincott Williams \& Wilkins.

[25] Yuan Liang, Weinan Song, JP Dym, Kun Wang, and Lei He. 2019. CompareNet: Anatomical Segmentation Network with Deep Non-local Label Fusion. In International Conference on Medical Image Computing and Computer-Assisted Intervention Springer, 292-300.

[26] Yuan Liang, Weinan Song, Jiawei Yang, Liang Qiu, Kun Wang, and Lei He. 2020 Atlas-aware ConvNet for Accurate yet Robust Anatomical Segmentation. In Asian Conference on Machine Learning. PMLR, 113-128.

[27] Yuan Liang, Weinan Song, Jiawei Yang, Liang Qiu, Kun Wang, and Lei He. 2020. X2Teeth: 3D Teeth Reconstruction from a Single Panoramic Radiograph. In International Conference on Medical Image Computing and Computer-Assisted Intervention. Springer, 400-409.

[28] Chen Lin, Junyi Gao, Hua Zheng, Jun Zhao, Hua Yang, Yue Zheng, Yihan Cao, Yufei Chen, Guoliang Wu, Guole Lin, et al. 2019. When to introduce threedimensional visualization technology into surgical residency: a randomized controlled trial. fournal of medical systems 43, 3 (2019), 71.

[29] Yue-Hin Loke, Francesco Capuano, Jason Mandell, Russell R Cross, Ileen Cronin, Paige Mass, Elias Balaras, and Laura J Olivieri. 2019. Abnormal Pulmonary Artery Bending Correlates With Increased Right Ventricular Afterload Following the Arterial Switch Operation. World fournal for Pediatric and Congenital Heart Surgery 10, 5 (2019), 572-581.

[30] Ting Luo, Changrong Shi, Xing Zhao, Yunsong Zhao, and Jinqiu Xu. 2016. Automatic synthesis of panoramic radiographs from dental cone beam computed tomography data. PloS one 11, 6 (2016), e0156976.
[31] Ian Mills, Julia Frost, Chris Cooper, David R Moles, and Elizabeth Kay. 2014. Patient-centred care in general dental practice-a systematic review of the literature. BMC Oral Health 14, 1 (2014), 64.

[32] Abu-Hussein Muhamad, Watted Nezar, and Abdulgani Azzaldeen. 2015. The curve of dental arch in normal occlusion. Open Science fournal of Clinical Medicine 3, 2 (2015), 47-54.

[33] SS Naumovich, SA Naumovich, and VG Goncharenko. 2015. Three-dimensional reconstruction of teeth and jaws based on segmentation of CT images using watershed transformation. Dentomaxillofacial Radiology 44, 4 (2015), 20140313.

[34] Kay T Oen, Analia Veitz-Keenan, Silvia Spivakovsky, Y Jo Wong, Eman Bakarman, and Julie Yip. 2014. CAD/CAM versus traditional indirect methods in the fabrication of inlays, onlays, and crowns. Cochrane Database of Systematic Reviews 2014, 4 (2014), CD011063.

[35] Laura J Olivieri, David Zurakowski, Karthik Ramakrishnan, Lillian Su, Fahad A Alfares, Matthew R Irwin, Jenna Heichel, Axel Krieger, and Dilip S Nath. 2018. Novel, 3D display of heart models in the postoperative care setting improves CICU caregiver confidence. World fournal for Pediatric and Congenital Heart Surgery 9, 2 (2018), 206-213.

[36] Floor MD Oosterink, Ad De Jongh, and Johan Hoogstraten. 2009. Prevalence of dental fear and phobia relative to other fear and phobia subtypes. European journal of oral sciences 117, 2 (2009), 135-143.

[37] A Prinz, M Bolz, and O Findl. 2005. Advantage of three dimensional animated teaching over traditional surgical videos for teaching ophthalmic surgery: a randomised study. British fournal of Ophthalmology 89, 11 (2005), 1495-1499.

[38] M Raja'a and Farzaneh Farid. 2016. Computer-based technologies in dentistry: types and applications. Journal of dentistry (Tehran, Iran) 13, 3 (2016), 215.

[39] Dianne Rekow. 1987. Computer-aided design and manufacturing in dentistry: a review of the state of the art. Fournal of Prosthetic Dentistry 58, 4 (1987), 512-516.

[40] R Gary Rozier, Alice M Horowitz, and Gary Podschun. 2011. Dentist-patient communication techniques used in the United States: the results of a national survey. The Journal of the American Dental Association 142, 5 (2011), 518-530.

[41] Richard M Satava. 1993. Virtual reality surgical simulator. Surgical endoscopy 7, 3 (1993), 203-205.

[42] Joseph D Shirk, David D Thiel, Eric M Wallen, Jennifer M Linehan, Wesley M White, Ketan K Badani, and James R Porter. 2019. Effect of 3-dimensional virtual reality models for surgical planning of robotic-assisted partial nephrectomy on surgical outcomes: a randomized clinical trial. JAMA network open 2, 9 (2019), e1911598-e1911598.

[43] Weinan Song, Yuan Liang, Kun Wang, and Lei He. 2020. Oral-3D: Reconstructing the 3D Bone Structure of Oral Cavity from 2D Panoramic X-ray. arXiv preprint arXiv:2003.08413 (2020)

[44] Moira A Stewart. 1995. Effective physician-patient communication and health outcomes: a review. CMAJ: Canadian medical association journal 152, 9 (1995), 1423.

[45] Joerg R Strub, E Dianne Rekow, and Siegbert Witkowski. 2006. Computeraided design and fabrication of dental restorations: current systems and future possibilities. The fournal of the American Dental Association 137, 9 (2006), 12891296.

[46] Emilia Taneva, Budi Kusnoto, and Carla A Evans. 2015. 3D scanning, imaging, and printing in orthodontics. Issues in contemporary orthodontics 148 (2015)

[47] Maxim Tatarchenko, Stephan R Richter, René Ranftl, Zhuwen Li, Vladlen Koltun, and Thomas Brox. 2019. What do single-view 3d reconstruction networks learn?. In Proceedings of the IEEE Conference on Computer Vision and Pattern Recognition. 3405-3414.

[48] John M Travaline, Robert Ruchinskas, and Gilbert E D’Alonzo Jr. 2005. Patientphysician communication: why and how. Journal of the American Osteopathic Association 105, 1 (2005), 13.

[49] Samantha A Welker. 2014. UROGYNECOLOGY PATIENT EDUCATION: VISUALIZING SURGICAL MANAGEMENT OF PELVIC ORGAN PROLAPSE. Ph.D. Dissertation. Johns Hopkins University.

[50] Eric Whaites and Nicholas Drage. 2013. Essentials of dental radiography and radiology. Elsevier Health Sciences.

[51] Rachel Marie Whitman and David Dufeau. 2017. Visualization of Cardiac Anatomy: New Approaches for Medical Education. The FASEB fournal 31, 1_supplement (2017), 736-8.

[52] Donna J Williams and Mubarak Shah. 1992. A fast algorithm for active contours and curvature estimation. CVGIP: Image understanding 55, 1 (1992), 14-26.

[53] Ke Xian, Chunhua Shen, Zhiguo Cao, Hao Lu, Yang Xiao, Ruibo Li, and Zhenbo Luo. 2018. Monocular relative depth perception with web stereo data supervision. In Proceedings of the IEEE Conference on Computer Vision and Pattern Recognition. 311-320.

[54] Xiaoqin Yang, Jing Hu, Songsong Zhu, Xinhua Liang, Jihua Li, and En Luo. 2011. Computer-assisted surgical planning and simulation for condylar reconstruction in patients with osteochondroma. British fournal of Oral and Maxillofacial Surgery 49, 3 (2011), 203-208 